\title{
EARLY RECOGNITION OF COGNITIVE ABILITY AND NUTRITIONAL MARKERS FOR DEMENTIA IN PARKINSON'S DISEASE
}

\author{
L. Håglin ${ }^{1}$, L. Bäckman ${ }^{1}$, J. Linder², L. Forsgren², M. Domellöf? 2,3
}

\begin{abstract}
Background: Cognitive decline and dementia are common non-motor problems in Parkinson's disease (PD). The underlying aetiology is multifaceted and both chronic and reversible causes for cognitive decline are likely to be present. Malnutrition is frequent in the Parkinson population, both early and late in the disease, and nutritional deficiencies could play a role in some cognitive deficits. Objectives: The objective is to study the association between nutritional status with focus on iron intake and homeostasis, mild cognitive impairment (MCI), and PD dementia (PDD). Setting and Participants: This study included 73 out of 145 patients with PD participating in a population-based study in northern Sweden. Measurements: Registration of nutritional status by laboratory analyses of blood plasma and neuropsychological assessments at time of diagnosis were performed. MCI and PDD were assessed yearly up to ten years after diagnosis. Mini Nutritional Assessments (Full-MNA score) and plasma variables detecting iron homeostasis were compared between patients with MCI and patients with normal cognition (NC). Motor severity was measured using the Unified Parkinson's disease rating scale III, (UPDRS III) and Hoehn and Yahr (H\&Y) staging scale. Cox proportional Hazard model were performed to see if any variables that differed between MCI and NC could predict PDD at follow-up. Results: Patients with MCI at time of diagnosis had lower levels of plasma iron (P-Fe) and albumin (P-Albumin) as well as a lower score on Full-MNA score. Dietary intake of iron was higher in patients with MCI than in patients with NC ( $\mathrm{p}=0.012)$. In logistic regression models adjusted for age, sex, and UPDRS III, lower levels of P-Fe ( $p=0.025)$ and P-Albumin $(\mathrm{p}=0.011)$ and higher dietary iron intake $(\mathrm{p}=0.019)$ were associated with MCI at baseline. A Cox regression model with dementia as endpoint revealed that lower levels of P-Fe increase the risk of dementia at follow-up with adjustments for age, sex, UPDRS III, and MCI at baseline (HR 95\% CI = 0.87 (0.78-0.98), p = 0.021). Conclusions: Low P-Fe was associated with cognitive disturbance at baseline and predicted dementia up to ten years after diagnosis in patients with PD. Low P-Albumin and malnutrition assessed with Full-MNA score were associated with MCI at baseline but did not predict dementia at follow-up.
\end{abstract}

Key words: Cognition, dementia, iron deficiency, Parkinson's disease.

\section{Introduction}

Parkinson's disease is a complex multifaceted disease with great variations in prognosis. Predicting the path the illness will take is next to impossible. Therefore, it is important to find predictors for different disease outcomes.

Cognitive decline and dementia are common nonmotor problems in PD and the incidence of dementia is two to six times higher than in the general population (1).

At diagnosis, $20-40 \%$ of the populations have Mild Cognitive Impairment (MCI) $(2-5)$. MCI at diagnosis increases the risk of Parkinson's disease dementia (PDD), but not all patients with MCI develop dementia

1. Department of Public Health and Clinical Medicine, Family Medicine, Umeå University, SE-901 87 Umeå, Sweden; 2. Department of Pharmacology and Clinical Neuroscience, Umeå University, SE-901 87 Umeå, Sweden; 3. Department of Psychology, Umeå University, SE-90187 Umeå Sweden

Corresponding Author: L Håglin, Department of Public Health and Clinical Medicine, Family Medicine, Umeå University, SE-901 87 Umeå, Sweden, lena. haglin@umu.se
(5). Different patterns of cognitive decline have been connected to PDD. For example, impairment of semantic fluency and pentagon copying has repeatedly been shown to predict PDD $(5,6)$. Low education level, postural instability, UPDRS (7), impaired olfactory function $(8,9)$, and male sex has been connected to higher rates of PDD (10).

Cognitive impairment has been reported among elderly with malnutrition and iron deficiency $(11,12)$. Solfrizzi et al. (2006) presented a review on the nutrientdementia risk pattern and suggested that more studies were needed to support the role of metals in progression of cognitive decline (13). A high iron intake has frequently been reported to increase the risk of PD, but no convincing evidence exists for an association between PDD and intake of iron. Cheng et al. (2011) reported that PD risk increased by 18\% (RR 1.18, $95 \%$ CI 1.02-1.37) by every $10-\mathrm{mg} /$ day increment in iron intake (14). Powers et al. (2003) reported that a high intake of iron, especially in combination with high manganese intake, may be related to an increased risk of PD (15). In addition, Powers et al. 
(2009) reported that high iron intake together with a low intake of cholesterol may be associated with an increased risk of PD (16). On the contrary, a higher intake of iron, magnesium, and zinc was independently associated with a reduced risk of PD (17). Follow-up of two large cohort studies, including accumulated information on nutrient intake, revealed a higher risk of PD with a high intake of non-heme iron in combination with low vitamin $C$ intake, while not with intake of heme iron (18).

This study investigated the association between nutritional status with a focus on iron intake and homeostasis in PD patients at time of diagnosis and risk of MCI and PDD at follow-up.

\section{Methods}

\section{Patients}

This community-based prospective study focuses on idiopathic forms of Parkinsonism in a catchment area (parts of Västerbotten county in northern Sweden) with 142000 inhabitants (19). All suspected cases with idiopathic Parkinsonism were referred to the neurological department that employed all the neurologists involved in this investigation. From January 2004 through April 2009, 185 cases with Parkinsonism were identified. In total 145 patients fulfilled diagnostic criteria for PD at the next follow-up (up to 10 years). Of these, 73 were included in the study (Table 1). Because the remaining 72 non-participating patients came to follow-up without fulfilling the three-day dietary registration, they were not included for a blood sample. Individuals not participating in the three-day dietary registration were older (mean 73.5 years vs mean 69.1 years, $p=0.006$ ) and scored higher on the UPDRS III (29.2 vs $24.7 \mathrm{p}=0.016)$ compared to those participating. There were no significant differences between the groups with regard to proportions of men and woman, total MMSE and MNA score.

A few patients refused to participate and were classified as drop outs. All participants were extensively examined during repeated visits the first month following initial contact. Information about demographics and disease history was obtained. All cases with suspected idiopathic Parkinsonism underwent a standardized clinical examination by a neurologist specializing in movement disorders.

To confirm the presence of PD, another specialist in movement disorders (blinded to the assessment of the previous examiner) evaluated a videotape of the patient undergoing the UPDRS III examination investigating PD related motor functions speech, facial expression, rigidity, tremor, posture, gait and bradykinesia (20). Patients were included if both examiners judged that they had fulfilled the clinical criteria for PD according to the UK Parkinson 's Disease Society Brain Bank (UK PDSBB) criteria. Motor severity was measured using the Unified Parkinson's disease rating scale III, (UPDRS III) and Hoehn and Yahr
(H\&Y) staging scale.

Table 1

Baseline variables (mean \pm sd) for the total PD group and for patients with Mild Cognitive Impairment (MCI) and Normal Cognition (NC). P-values as a result from comparing variables between $\mathrm{MCI}$ and $\mathrm{NC}$

\begin{tabular}{lllll}
\hline Variables & $\begin{array}{l}\text { PD patients } \\
\mathbf{n}=\mathbf{7 3}\end{array}$ & $\begin{array}{l}\text { MCI } \\
\mathbf{n = 3 0}\end{array}$ & $\begin{array}{l}\text { NC } \\
\mathbf{n}=\mathbf{4 3}\end{array}$ & p-value \\
\hline Age, years & $69.0 \pm 8.7$ & $72.2 \pm 8.2$ & $66.8 \pm 8.4$ & 0.008 \\
male/female n & $38 / 35$ & $20 / 10$ & $18 / 25$ & 0.037 \\
Education, years & $10.4 \pm 4.4$ & $8.7 \pm 3.5$ & $11.5 \pm 4.7$ & 0.009 \\
UPDRS-III score & $24.6 \pm 10.7$ & $28.2 \pm 9.1$ & $22.1 \pm 10.9$ & 0.016 \\
H\&Y & 2.0 & 2.0 & 2.0 & 0.107 \\
ADL & $88.9 \pm 5.5$ & $86.9 \pm 6.0$ & $91.7 \pm 3.7$ & 0.001 \\
PIGD/Ind/TD & $38 / 13 / 22$ & $18 / 2 / 10$ & $20 / 11 / 12$ & 0.114 \\
\hline
\end{tabular}

Abbreviations: UPDRS = Unified Parkinson's Disease Rating Scale; $\mathrm{H} \& \mathrm{Y}=$ Hoehn \& Yahr stage, median; $\mathrm{ADL}=$ Activity of daily living; PIGD $=$ Postural instability Gait Disorder; Ind = Indeterminate; TD = Tremor dominance:

The UPDRS scoring was performed with patients on their regular medication when started on dopaminergic treatment. The motor subtype was classified as tremor dominant (TD), postural instability and gait difficulty (PIGD) or indeterminate (Ind) according to Jancovic et al (21). The MMSE was used as a screening instrument for global cognition.

The study was approved by the Regional Medical Ethics Board in Umeå, Sweden. Written informed consent was obtained from all participants.

\section{Medication for PD}

The levodopa equivalent daily dose (LEDD) was calculated at baseline and at follow-ups by use of a conversion factor for each of the anti-Parkinson medications (22). Information about LEDD at baseline revealed that only two patients had started antiParkinson treatment at time of diagnosis. At first follow-up (six months), all but nine patients had started treatment and about $50 \%$ of the patients had a LEDD $<200$ and $8 \%$ had $>300$. Medication was introduced over the first weeks after diagnosis. The nutritional investigation, including the blood sampling, was performed in a non-standardized manner according to drug therapy during this time. Thirteen patients started anti-Parkinson medication before the blood sampling.

\section{Nutritional assessments}

The Full Mini-Nutritional Assessment (MNA score), an international validated screening tool, was used to assess nutritional status and performed by the dietician 
concomitantly with anthropometrical measurements. The screening consists of 18 questions regarding anthropometry, diet, and health (23). MNA total scores between 24 and 30 points indicate optimal nutritional status and scores between 17 and 23.5 points indicate at risk for malnutrition.

Dietary data were collected from a three-day dietary registration (3-day DR) performed by the patients three days before a scheduled visit to the Department of Neurology. The subjects recorded in a protocol what they ate and drank during those three days. To describe portion sizes, they had access to a booklet with photographs and drawings of various foods and dishes. Standard household measures or package sizes were used for food items not included. Energy and nutrient intake were calculated using the dietary software program Dietist XP (Kost och näringsdata, Stockholm, Sweden), which employs the National Food Administrations food database (NFA database 2.00, Uppsala, Sweden).

\section{Biochemical analysis}

Blood samples at baseline were collected at admittance between 8 am and $4 \mathrm{pm}$ in a non-fasting condition on the day when the patients were admitted for assessments of nutritional status. Plasma samples were collected and stored at $-70{ }^{\circ} \mathrm{C}$ until iron, ferritin, albumin, and transferrin were measured. Transferrin saturation was calculated S-Fe $[\mu \mathrm{mol} / \mathrm{L}]$ x $100 /$ (S-Transferrin $[\mathrm{g} / \mathrm{L}]$ x 25.1) (Trf \%). Analysis was performed in clinical routine in the accredited laboratory at Umeå University Hospital. Plasma levels of albumin and transferrin were analysed by Vitros PHOS Slides, Vitros ALB Slides, and Vitros TRFRN reagent on an Ortho Vitros 5.1 FS analyser.

\section{Cognition}

A battery of neuropsychological tests was performed and used for MCI and PDD classification at baseline, one year, three years, five, eight, and ten years. For MCI classification, the MDS Task force guidelines were used (24). Since the battery of tests included a minimum of two tests in all domains except language, modified level II criteria were applied (25). For patients not performing the full neuropsychological testing, MCI classification was based on self-perceived cognitive decline and MMSE (cutoff $\leq 29$ ) (26). All but four patients with blood samples performed the neuropsychological evaluation at baseline. PDD was diagnosed according to published criteria by neurologists experienced in neurodegenerative disorders (27). In periods between the neuropsychological testing, PDD was diagnosed by decline in MMSE, decline in basic activities of daily living (ADL) (28) due to decreased cognition, and cognitive decline reported from patients and/or family member. In addition to MCI and PDD, composite scores for different cognitive domains (executive function, attention, language, visuospatial function, and episodic memory) were calculated based on means of the $\mathrm{z}$ scores for each individual test. Neuropsychological measures used for classification of Mild Cognitive Impairment and calculation of composite scores. Pentagon copying was not included in the calculation of composite scores.

Episodic memory: Free and Cued Selective Reminding Test (FCSRT) free recall, Brief Visuospatial Memory Test (BVMT) total, Brief Visuospatial Memory Test (BVMT) delayed.

Visuospatial function: Benton Judgement of Line Orientation (BJOLOT), pentagon copying from MMSE. Language function: Boston Naming Test. Working memory and attention: Digit span backwards, Trail Making Test part B. Executive function: Wisconsin Card Sorting Test (WCST) total errors, Wisconsin Card Sorting Test (WCST) preservative errors, Semantic fluency (animals in 60 seconds).

\section{Data analyses}

The results are presented as mean and standard deviation with min-max for each variable presented. Independent two-tailed tests were used to compare patients with MCI and NC. For non-normal distributed data and variables with skewed distribution, nonparametric tests were performed: the Mann-Whitney and the Wilcoxon Signed Ranks Test. Most variables were approximately normally distributed except for H\&Y score, P-Ferritin, and dietary iron intake. Because of the non-normal distribution, P-Ferritin and dietary intake of iron were dichotomized by the median value (P-Ferritin median $=128.7 \mu \mathrm{g} / \mathrm{L}$ and dietary iron intake median $=$ $9.77 \mathrm{mg} /$ day).

To check whether the variables that significantly differed between MCI and NC independent of age, sex, and UPDRS III, logistic regression models adjusted for age, sex, and UPDRS III were performed. To investigate the specific cognitive domains (i.e., the association with the variables differing between patients with MCI and patients with NC), partial correlations was used with corrections for age, sex, and UPDRS III with the biochemical assessments and merged scores for the separate cognitive domains.

The Cox proportional hazards model was used to study the association between biochemical assessments at baseline and the development of PDD. Variables associated with MCI were included in Cox-regression models, first univariate, and then multivariate adjusted for age, gender, UPDRS III at baseline, and finally MCI at baseline was added.

The program Predictive Analytics Software (SPSS Statistics, version 24 SPSS Inc., Chicago IL, USA) was used for the analysis. 
Table 2

Baseline variables (mean \pm sd) used to assess iron status in PD patients with Mild Cognitive Impairment (MCI) and Normal Cognition (NC). The level of significance from the logistic regression with adjustments for age, sex, and UPDRS III are presented in the right column

\begin{tabular}{|c|c|c|c|c|}
\hline $\begin{array}{l}\text { Variables (reference values } \\
\text { or recommended) }\end{array}$ & MCI $n=30$ & $\mathrm{NC} n=43$ & P-value & $\begin{array}{l}P-v \text { al u e } \\
\text { adj. }\end{array}$ \\
\hline $\mathrm{P}-\mathrm{Fe}, \mu \mathrm{mol} / \mathrm{L}(9-34)$ & $16.6 \pm 4.3$ & $18.5 \pm 5.0$ & 0.088 & 0.025 \\
\hline $\operatorname{TrF} \%(\mathrm{~F}=15-50, \mathrm{M}=15-60)$ & $30.8 \pm 9.5$ & $33.1 \pm 9.9$ & 0.345 & 0.154 \\
\hline $\begin{array}{l}\text { P-Ferritin, } \mu \mathrm{g} / \mathrm{L} \text { (>50 yrs, } \\
30-400 \text { ) }\end{array}$ & $182 \pm 201$ & $184 \pm 195$ & 0.973 & 0.488 \\
\hline P-Ferritin* & $15 / 15$ & $21 / 22$ & 0.922 & 0.646 \\
\hline $\begin{array}{l}\text { P-Transferrin, g/L (1.87- } \\
3.19)\end{array}$ & $2.2 \pm 0.4$ & $2.3 \pm 0.3$ & 0.340 & 0.214 \\
\hline $\begin{array}{l}\text { P-Albumin, g/L } 40-70 \text { yrs } \\
36-45>70 \text { yrs } 34-45\end{array}$ & $39.1 \pm 3.4$ & $42.0 \pm 3.5$ & 0.001 & 0.011 \\
\hline MNA - total (24-30) & $24.9 \pm 2.9$ & $26.1 \pm 1.9$ & 0.035 & 0.057 \\
\hline $\begin{array}{l}\text { Dietary iron intake, } \\
\text { Rec intake per } / \text { day }=9\end{array}$ & $19 / 10$ & $14 / 26$ & 0.068 & 0.101 \\
\hline Dietary iron intake mg/day* & $12.1 \pm 7.2$ & $9.7 \pm 3.6$ & 0.012 & 0.019 \\
\hline
\end{tabular}

* Plasma ferritin and dietary iron intake were dichotomized to over and under median value (median $=128.7 \mu \mathrm{g} / \mathrm{L}$ and median $=9.77 \mathrm{mg} /$ day respectively) As seen in table 3. Abbreviations: $\operatorname{TrF} \%=\mathrm{S}-\mathrm{Fe}[\mu \mathrm{mol} / \mathrm{L}] \times 100 /$ (S-Transferrin [g/L] x 25.1); MNA = Mini Nutritional Assessment.

\section{Results}

\section{Baseline data}

Non-motor and motor functions assessed at baseline for patients with PD $(n=73)$ and for patients with MCI $(n=30)$ and NC $(n=43)$ are presented in Table 1. At baseline, MCI was more prevalent in males than in females and was associated with older age, lower level of education, and higher UPDRS III score. Patients with MCI had higher daily intake of iron and lower MNA score, P-Albumin, and P-Fe before adjusting for age, sex, and UPDRS III (Table 2). After adjusting, the OR for having MCI was still significant for P-Albumin, P- Fe and daily intake of iron but not for MNA score (Exp B: 95\% CI = 0.799 (0.672-0.950), $\mathrm{p}=0.011), \mathrm{P}-\mathrm{Fe}(\operatorname{Exp} \mathrm{B}: 95 \% \mathrm{CI}=0.854$ (0.744-0.980), $\mathrm{p}=0.025)$, dietary iron intake (Exp B: 95\% $\mathrm{CI}=4.425(1.54-18.9) \mathrm{p}=0.019)$, and MNA score (Exp B: $95 \% \mathrm{CI}=0.79$ (0.984-1.125, $\mathrm{p}=0,057$, respectively) (Table 2 ). Biochemical and nutritional assessments for males and females, including variables for iron homeostasis, are presented in Table 3. Males had higher P-Ferritin and dietary iron intake than females. No other differences indicate sex dependent disturbed iron homeostasis and nutritional status.

\section{Correlations between P-Albumin and neuropsychological tests}

Composite scores of working memory correlated with P-Albumin levels $(\mathrm{r}=0.452, \mathrm{p}<0.001)$ and P-Ferritin levels $(r=270, p=0.025)$. Both correlations were stronger for males than females: P-Albumin (male; $\mathrm{r}=0.549, \mathrm{p}<$ 0.001 and female $\mathrm{r}=0.331, \mathrm{p}=0.069$ (Figure 1), P-Ferritin (male; $r=0.413, p=0.01$ and female $r=0.212, p=0.252$ ) Composite scores of episodic memory correlated with P-albumin $(\mathrm{r}=0.253, \mathrm{p}=0.036)$. After adjusting for age, gender, and UPDRS III score in partial correlations, the positive association between composite score of working memory and P-Albumin $(\mathrm{r}=0.346, \mathrm{p}=0.004)$ and P-Ferritin $(r=0.309, p=0.012)$ remained significant.

Table 3

Baseline variables in mean \pm sd and for min-max used to assess iron status in patients with PD

\begin{tabular}{llll}
\hline $\begin{array}{l}\text { Variables (reference values or } \\
\text { recommended) }\end{array}$ & Males n=39 & Females n=34 & P-value \\
\hline P-Fe, $\mu$ mol/L & $18.2 \pm 4.6$ & $17.1 \pm 4.9$ & 0.304 \\
(9-34) & $8.2-28.3$ & $8.0-30.5$ & \\
TrF, \% & $33.9 \pm 10.0$ & $30.1 \pm 9.2$ & 0.208 \\
(F=15-50, M=15-60) & $12-56$ & $14-54$ & \\
P-Ferritin, $\mu \mathrm{g} / \mathrm{L}$ & $240 \pm 247$ & $118 \pm 76$ & 0.005 \\
(>50 yrs, 30-400) & $12-1008$ & $15-307$ & \\
P-Ferritin * & $23 / 16$ & $13 / 21$ & 0.077 \\
P-Transferrin, g/L & $2.2 \pm 0.34$ & $2.3 \pm 0.34$ & 0.270 \\
(1.87-3.19) & $1.56-2.96$ & $1.70-3.59$ & 0.652 \\
P-Albumin, g/L & $40.6 \pm 3.8$ & $41.0 \pm 3.7$ & \\
40-70 yrs 36-45 & $32-49$ & $34-49$ & \\
$>70$ yrs 34-45 & & & \\
MNA - total & $26.0 \pm 2.5$ & $25.2 \pm 2.5$ & 0.224 \\
(24-30) & $21.0-30.0$ & $17.5-29.0$ & \\
Dietary iron intake, mg/day & $11.9 \pm 6.5$ & $9.3 \pm 3.4$ & \\
(Rec intake per/day = 9) & $4.2-37.0$ & $5.0-19.9$ & \\
Dietary iron intake* & $24 / 14$ & $9 / 22$ & \\
\hline$*$ Plasma fert & & & \\
\hline
\end{tabular}

* Plasma ferritin and dietary iron intake were dichotomized to over and under median value (median $=128.7 \mu \mathrm{g} / \mathrm{L}$ and median $=9.77 \mathrm{mg} /$ day respectively); Abbreviations: $\operatorname{TrF} \%=$ S-Fe $[\mu \mathrm{mol} / \mathrm{L}] \times 100$ / (S-Transferrin $[\mathrm{g} / \mathrm{L}] \times 25.1$ ); MNA $=$ Mini Nutritional Assessment.

\section{Cox regressions in prediction of $P D D$}

Results from Cox regression analysis are shown in Table 4. Lower baseline P-Fe $(\mu \mathrm{mol} / \mathrm{L})$ and P-Albumin $(\mathrm{g} / \mathrm{L})$ levels increased the risk of PDD at follow-up in univariate models. In models controlling for age, sex, and UPDRS III, lower levels of P-Fe significantly increased the risk of PDD (HR $(95 \% \mathrm{CI})=0.85(0.76-0.94), \mathrm{p}=0.002)$. Adding $\mathrm{MCI}$ to the model did not affect the higher risk of PDD with lower P-Fe levels (HR $(95 \% \mathrm{CI})=0.87(0.78$ $0.98), \mathrm{p}=0.02$ ). 


\section{Discussion}

The main finding from our study is that low levels of $\mathrm{P}-\mathrm{Fe}$ were associated with $\mathrm{MCI}$ at time of diagnosis and increased risk of dementia over 10 years, while neither intake of iron, MNA-score, nor P-Albumin predicted dementia at follow-up. In addition, significantly lower levels of P-Albumin and lower score on Full MNA, indicating risk of malnutrition, and higher dietary intake of iron were seen in patients with PD-MCI at time of diagnosis. With adjustments for age, sex, and UPDRS III, the difference was maintained only for P-Albumin and dietary intake of iron.

\section{Figure 1}

P-Albumin levels correlated with composite score of working memory and were stronger for males than females (male: $r=0.549, p<0.001$ and female: $r=0.336, p$

$$
=0.064)
$$

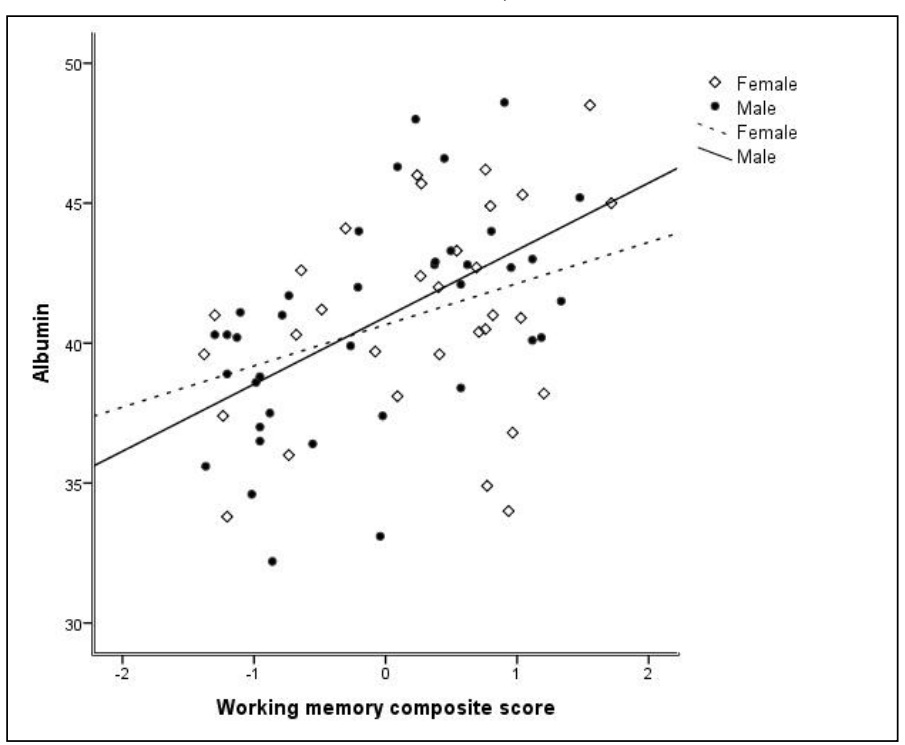

Lower levels of P-Albumin, P-Fe, and Full MNA score at diagnosis for patients with $\mathrm{MCI}$ indicate malnutrition, which may have had its origin years prior to diagnosis and first symptoms. An association between MNA score and MCI in older in-patients (29) and between MNA score and disease severity and progress of disability in patients with PD has been reported $(30,31)$. Nutritional status may be affected by several of the disease-related measurements such as ADL, UPDRS, and medication.

Used at baseline to assess malnutrition, the MNA score was lower in patients with MCI compared to patients with NC, but it did not predict PDD at followup. Different components of nutritional status besides results from a screening test may have importance when focusing on risk of dementia progression (32). However, MNA score has been shown to correlate with P-Albumin and dietary intake of iron (33). Being malnourished and having MCI may also have contributed to low P-Fe levels and may be one of several components of nutritional status that can influence progress to PDD. Difficulties in eating abilities and cutting food, often reported in PD patients, can result in a low intake of nutrients and energy. Age, disease, and sex-related factors also contribute to the consequences of malnutrition. In addition to finding predictors for different disease outcomes at the time of diagnosis, we also need to focus on finding PDD predictors already in the prodromal phase of PD.

In the present study, P-Albumin was significantly lower in patients with $\mathrm{MCI}$ than in patients with intact cognition at baseline. Albumin has previously mostly been associated with mortality and aging $(34,35,36)$, although a handful studies have found an association of low levels of P-Albumin with cognitive decline $(37,38)$. The different P-Albumin levels between MCI and NC were still significant after controlling for age and disease severity, which indicates that P-Albumin has a specific connection to cognitive decline. Also, the correlation between P-Albumin and P-Ferritin within normal limits and working memory was stronger in men than in woman, which suggests a sex difference for the impact of P-Albumin and P-Ferritin levels on working memory. No studies have reported this association before. As several comparisons were performed, the results may be spurious. Had we applied Bonferroni correction, only

Table 4

Results from Cox regression ( $\mathrm{HR}$ 95\% CI) with Dementia as outcome variable and nutritional variables as explanatory included are presented with bivariate and multivariate calculations

\begin{tabular}{|c|c|c|c|c|c|c|}
\hline & \multicolumn{2}{|c|}{ Univariate } & \multicolumn{2}{|c|}{ Multivariate $^{*}$} & \multicolumn{2}{|c|}{ Multivariate $^{* *}$} \\
\hline & HR $(95 \%$ CI) & P-value & HR $(95 \% \mathrm{CI})$ & P-value & HR $(95 \% \mathrm{CI})$ & P-value \\
\hline \multicolumn{7}{|l|}{ Total $n=72$} \\
\hline $\mathrm{P}-\mathrm{Fe}, \mu \mathrm{mol} / 1$ & $0.91(0.83-0.99)$ & 0.038 & $0.85(0.76-0.94)$ & 0.002 & $0.87(0.78-0.98)$ & 0.021 \\
\hline P-Albumin, g/L & $0.88(0.80-0.98)$ & 0.015 & $0.91(0.81-1.02)$ & 0.107 & $0.98(0.85-1.12)$ & 0.742 \\
\hline MNA-total score & $0.92(0.80-1.05)$ & 0.199 & $0.96(0.83-1.12)$ & 0.601 & $1.03(0.87-1.19)$ & 0.735 \\
\hline Mean dietary iron intake $>9.77 \mathrm{mg} /$ day & $1.31(0.60-2.83)$ & 0.498 & $1.26(0.53-3.02)$ & 0.600 & $1.12(0.44-2.85)$ & 0.805 \\
\hline
\end{tabular}

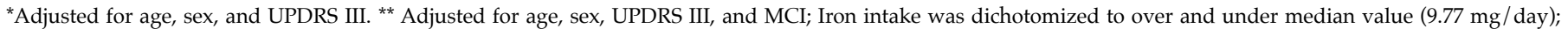
Abbreviations: MNA = Mini Nutritional Assessment. 
P-Albumin would have been considered significantly different between MCI and NC. Frangos et al. (2016) concluded that both age and disease contribute to low albumin, which could explain low haemoglobin levels in malnourished associated anaemia in very old hospitalized elderly (39). The baseline levels of P-Albumin and MNA score indicate an early association with cognitive decline in patients with PD in our study population.

Haemoglobin analysis was not performed on the same occasion/visit with blood sampling and assessment of malnutrition, limiting the possibility to assess anaemia. However, iron deficiency may be important for cognitive function independent from anaemia. Yavuz et al. (2012) studied geriatric patients (mean 72 years of age) and concluded that iron levels and Trf \% independently correlated with MMSE. In that study, patients with dementia had low Trf \% and a low MMSE associated with iron deficiency (11). Mean levels for P-Transferrin increase to compensate for low P-Fe and increased need, but in our study mean levels for P-Transferrin decreased with malnutrition. P-Fe is transported with transferrin, and about $30-40 \%$ of this capacity is used dependent on age and sex and increases after the age of 70 years $(40,41)$. Besides being an iron transporter, P-Transferrin is a marker for nutritional status $(42,43)$. By a shorter halflife than albumin, transferrin may be a strong marker for protein energy malnutrition within days to weeks.

Iron deficiency may affect dopamine metabolism $(44,45)$, and dysregulation of iron metabolism has been described as a risk factor for PD (46). Iron homeostasis is reported to be important for cognition (47). Cognitive impairment has been reported among elderly with iron deficiency and malnutrition (11,12). Iron deficiency in adults and elderly indicates that age and sex may have contributed to hypo-metabolism that might have resulted in early dementia in Parkinson's disease (48). In the present study, age was associated with both MCI at baseline and with increased risk for dementia over 10 years. The vicious circle with ageing, malnutrition, and cognitive decline may be even stronger in PD patients due to disease-related disturbances than in a non-PD elderly population. A low bioavailability of iron in the intestine (the main cause of iron deficiency, at least among elderly) could have generated the low $\mathrm{P}-\mathrm{Fe}$ in the present population of patients, for example, due to gastrointestinal disturbances like gastro pares and achlorhydria (49). The level of P-Fe varies from day to day and low levels indicate either low intake or malabsorption. The risk of MCI, associated with either high intake of iron or low plasma $\mathrm{Fe}$, indicate the importance of intestinal function for maintaining iron homeostasis. A high intake of iron may affect bioavailability of other minerals and thereby contribute to deficit cognition. To study influence from dietary intake, more information on nutrient density in general and individual energy intake are needed together with data on intestinal function and medication. Reporting dietary intake may also have been affected by the level of cognitive ability.

\section{Conclusion}

Our results indicate that low P-Fe was associated with $\mathrm{MCI}$ at baseline and increases risk for dementia up to 10 years after diagnosis of PD. Low P-Albumin and disturbed cognition in PD may influence the progression to dementia and be a sign of protein energy malnutrition. High intake of iron increased the risk of MCI at baseline but did not show any effect on PDD risk. We suggest that low P-Fe in our sample might be related to a disturbed regulation, including intestinal absorption in iron metabolism as well as malnutrition.

Funding: This study was funded by The Swedish Parkinson Foundation (Svenska Parkinsonstiftelsen), Swedish Parkinson foundation, Neuro Sweden, The Swedish Parkinson Disease Association, The Swedish Medical Research Council and Västerbotten County Council (ALF).

\section{Conflict of interest: The authors declared that they had no conflict of interests.}

Ethical Standard: The study was approved by the Ethics Committee of the Faculty of Medicine at Umeå University. Written, informed consent was obtained from all participants.

\section{References}

1. Caballol N, Martí MJ, Tolosa E. Cognitive dysfunction and dementia in Parkinson disease. Mov Disord 2007;22 Suppl 17:S358-66.

2. Muslimovic D, Post B, Speelman JD, Schmand B. Cognitive profile of patients with newly diagnosed Parkinson disease. Neurology 2005;65(8):1239-1245.

3. Aarsland D, Brønnick K, Larsen JP, Tysnes OB, Alves G; Norwegian ParkWest Study Group. Cognitive impairment in incident, untreated Parkinson disease: the Norwegian ParkWest study. Neurology 2009 Mar 31;72(13):1121-1126.

4. Foltynie T, Brayne CE, Robbins TW, Barker RA. The cognitive ability of an incident cohort of Parkinson's patients in the UK. The CamPaIGN study. Brain 2004;127(Pt 3):550-560.

5. Domellöf ME, Ekman U, Forsgren L, Elgh E. Cognitive function in the early phase of Parkinson's disease, a five-year follow-up. Acta Neurol Scand 2015;132(2):79-88

6. Williams-Gray CH, Mason SL, Evans JR, Foltynie T, Brayne C, Robbins TW Barker RA. The CamPaIGN study of Parkinson's disease: 10-year outlook in an incident population-based cohort. J Neurol Neurosurg Psychiatry 2013;84(11):1258-1264.

7. Vasconcellos LF, Pereira JS. Parkinson's disease dementia: Diagnostic criteria and risk factor review. J Clin Exp Neuropsychol 2015;37(9):988-993.

8. Domellöf ME, Lundin KF, Edström M, Forsgren L. Olfactory dysfunction and dementia in newly diagnosed patients with Parkinson's disease. Parkinsonism Relat Disord 2017;38:41-47.

9. Hu Y, Ding WT, Zhu XN, Wang XL. A mini review: Tau transgenic mouse models and olfactory dysfunction in Alzheimer's Disease. Zhongguo Ying Yong Sheng Li Xue Za Zhi 2015;31(6):481-490.

10. Cereda E, Cilia R, Klersy C, Siri C, Pozzi B, Reali E, et al. Dementia in Parkinson's disease: Is male gender a risk factor? Parkinsonism Relat Disord 2016;26:67-72.

11. Yavuz BB, Cankurtaran M, Haznedaroglu IC, Halil M, Ulger Z, Altun B, et al. Iron deficiency can cause cognitive impairment in geriatric patients. J Nutr Health Aging 2012;16(3):220-224.

12. Khater MS, Abouelezz NF. Nutritional status in older adults with mild cognitive impairment living in elderly homes in Cairo, Egypt. J Nutr Health Aging 2011;15(2):104-108.

13. Solfrizzi V, Colacicco AM, D'Introno A, Capurso C, Parigi AD, Capurso SA et al. Macronutrients, aluminium from drinking water and foods, and other metals in cognitive decline and dementia. J Alzheimers Dis 2006;10(2-3):303330.

14. Cheng P, Yu J, Huang W, Bai S, Zhu X, Qi Z, et al. Dietary intake of iron, zinc, copper, and risk of Parkinson's disease: a meta-analysis. Neurol Sci 2015;36(12):2269-2275.

15. Powers KM, Smith-Weller T, Franklin GM, Longstreth WT Jr, Swanson PD, Checkoway H. Parkinson's disease risks associated with dietary iron, manganese, and other nutrient intakes. Neurology 2003 Jun 10;60(11):17611766. 
16. Powers KM, Smith-Weller T, Franklin GM, Longstreth WT Jr, Swanson PD, Checkoway H. Dietary fats, cholesterol and iron as risk factors for Parkinson's disease. Parkinsonism Relat Disord 2009 Jan;15(1):47-52.

17. Miyake Y, Tanaka K, Fukushima W, Sasaki S, Kiyohara C, Tsuboi Y, et al. Dietary intake of metals and risk of Parkinson's disease: a case-control study in Japan. J Neurol Sci 2011:15;306(1-2):98-102.

18. Logroscino G, Gao X, Chen H, Wing A, Ascherio A. Dietary iron intake and risk of Parkinson's disease. Am J Epidemiol 2008 Dec 15;168(12):1381-1388.

19. Linder J, Stenlund H, Forsgren L. Incidence of Parkinson's disease and parkinsonism in northern Sweden: a population-based study. Mov Disord 2010;25(3):341-348.

20. Fahn S, Elton RL, the UPDRS Development Committee. Unified Parkinson's disease rating scale. In: Fahn S, Marsden CD, Calne D, Goldstein M, Eds. Recent developments in Parkinson's disease. Florham Park, NJ: Macmillan Healthcare Information; 1987: 153-64.

21. Jankovic J, McDermott M, Carter J, Gauthier S, Goetz C, Golbe L, et al. Variable expression of Parkinson's disease: a base-line analysis with quality of life in the general population. Mov Disord 2008;23:790-796.

22. Tomlinson CL, Stowe R, Patel S, Rick C, Gray R, Clarke CE. Systematic review of levodopa dose equivalency reporting in Parkinson's disease. Mov Disord 2010;25(15):2649-2653.

23. Guigoz Y. The Mini Nutritional Assessment (MNA) review of the literatureWhat does it tell us? J Nutr Health Aging 2006;10(6):466-485; discussion 485487.

24. Litvan I, J.G. Goldman, A.I. Troster, B.A. Schmand, D. Weintraub, R.C. Petersen,B, et al. Diagnostic criteria for mild cognitive impairment in Parkinson's disease: movement Disorder Society Task Force guidelines, Mov Disord 2012;27:349e356.

25. Yarnall, D.P. Breen, G.W. Duncan, T.K. Khoo, S.Y. Coleman, M.J. Firbank, C. et al. Characterizing mild cognitive impairment in incident Parkinson disease: the ICICLE-PD study, Neurology 2014;82:308e316

26. Hoops S, Nazem S, Siderowf AD, Duda JE, Xie SX, Stern MB, et al.Validity of the MoCA and MMSE in the detection of MCI and dementia in Parkinson disease. Neurology 2009;73(21):1738-1745.

27. Emre M, Aarsland D, Brown R, Burn DJ, Duyckaerts C, Mizuno Y, et al Clinical diagnostic criteria for dementia associated with Parkinson's disease. Mov Disord 2007;22(12):1689-1707; quiz 1837. Review

28. Schwab RS, England AC, Jr. Projection technique for evaluating surgery in Parkinson's disease. In: Gillingham FJ, Donaldson IML, eds. Third symposium on Parkinson's disease. Edinburgh and London: E\&S Livingston Ltd, 1969;152-157.

29. Orsitto G. Different components of nutritional status in older inpatients with cognitive impairment. J Nutr Health Aging 2012;16(5):468-471.

30. Shidfar F, Babaii Darabkhani P, Yazdanpanah L, Karkheiran S, NoorollahiMoghaddam H, Haghani H. Assessment of nutritional status in patients with Parkinson's disease and its relationship with severity of the disease. Med J Islam Repub Iran 2016;30:454. eCollection 2016.

31. Tomic S, Pekic V, Popijac Z, Pucic T, Petek M, Kuric TG, et al. What increases the risk of malnutrition in Parkinson's disease? J Neurol Sci 2017;375:235-238.

32. Sanders C, Behrens S, Schwartz S, Wengreen H, Corcoran CD, Lyketsos CG, et al. Nutritional Status is associated with Faster Cognitive Decline and Worse
Functional Impairment in the Progression of Dementia: The Cache County Dementia Progression Study1. J Alzheimers Dis 2016;52(1):33-42.

33. Vellas B, Guigoz Y, Baumgartner M, Garry PJ, Lauque S, Albarede JL. Relationships between nutritional markers and the mini-nutritional assessment in 155 older persons. J Am Geriatr Soc 2000;48(10):1300-1309.

34. Aptaker RL, Roth EJ, Reichhardt G, Duerden ME, Levy CE. Serum albumin level as a predictor of geriatric stroke rehabilitation outcome. Arch Phys Med Rehabil 1994;75(1):80-84.

35. D'Erasmo E, Pisani D, Ragno A, Romagnoli S, Spagna G, Acca M. Serum albumin level at admission: mortality and clinical outcome in geriatric patients. Am J Med Sci 1997;314(1):17-20.

36. Domellöf ME, Ekman U, Forsgren L, Elgh E. Cognitive function in the early phase of Parkinson's disease, a five-year follow-up. Acta Neurologica Scandinavica 2015;132(2):79-88

37. Gom I, Fukushima H, Shiraki M, Miwa Y, Ando T, Takai K, et al. Relationship between serum albumin level and aging in community-dwelling selfsupported elderly population. J Nutr Sci Vitaminol (Tokyo). 2007;53(1):37-42.

38. La Rue A, Koehler KM, Wayne SJ, Chiulli SJ, Haaland KY, Garry PJ. Nutritional status and cognitive functioning in a normally aging sample: a 6-y reassessment. Am J Clin Nutr 1997;65(1):20-29.

39. Ng TP, Niti M, Feng L, Kua EH, Yap KB. Albumin, apolipoprotein E-epsilon4 and cognitive decline in community-dwelling Chinese older adults. J Am Geriatr Soc 2009;57(1):101-106.

40. Frangos E, Trombetti A, Graf CE, Lachat V, Samaras N, Vischer UM, et al Malnutrition in Very Old Hospitalized Patients: A New Etiologic Factor of Anemia? J Nutr Health Aging 2016;20(7):705-713.

41. Muñoz M, García-Erce JA, Remacha AF. Disorders of iron metabolism. Part 1: molecular basis of iron homoeostasis. J Clin Pathol 2011;64(4):281-286.

42. Waldvogel-Abramowski S, Waeber G, Gassner C, Buser A, Frey BM, Favrat B, et al. Physiology of iron metabolism. Transfus Med Hemother 2014 Jun;41(3):213-21.

43. Håglin L, Bäckman L. Covariation between plasma phosphate and daytime cortisol in early Parkinson's disease. Brain Behav 2016;6(12):e00556.

44. Belaidi AA, Bush AI. Iron neurochemistry in Alzheimer's disease and Parkinson's disease: targets for therapeutics. J Neurochem 2016 Oct;139 Suppl 1:179-197.

45. Ramsey AJ, Hillas PJ, Fitzpatrick PF. Characterization of the active site iron in tyrosine hydroxylase. Redox states of the iron. J Biol Chem 1996;271(40):24395-24400.

46. Logroscino G, Chen H, Wing A, Ascherio A. Blood donations, iron stores, and risk of Parkinson's disease. Mov Disord 2006;21(6):835-838.

47. Murray-Kolb LE, Beard JL Iron treatment normalizes cognitive functioning in young women. Am J Clin Nutr 2007;85(3):778-787.

48. Baba T, Hosokai Y, Nishio Y, Kikuchi A, Hirayama K, Suzuki K, et al. Longitudinal study of cognitive and cerebral metabolic changes in Parkinson's disease. J Neurol Sci 2017;372:288-293.

49. Bhutto A, Morley JE. The clinical significance of gastrointestinal changes with aging. Curr Opin Clin Nutr Metab Care. 2008 Sep;11(5):651-660. 